\title{
HONGOS SIMBIONTES DE HORMIGAS CORTADORAS DE HOJAS DEL GÉNERO ACROMYRMEX EN BOSQUES SECOS DEL CENTRO DE ARGENTINA
}

\author{
David Pelissero $^{1}$ (D), Francisco Kuhar ${ }^{1,2}$ (iD, Mariana Pereyra ${ }^{1}$ (iD) \& Eduardo Nouhra ${ }^{1,3}$ (iD
}

\begin{abstract}
${ }^{1}$ Instituto Multidisciplinario de Biología Vegetal (CONICET-UNC), Universidad Nacional de Córdoba, Av. Vélez Sarsfield 1666, X5016GCN Córdoba, Córdoba, Argentina; davidpelissero@mi.unc.edu.ar (autor corresponsal).

${ }^{2}$ Facultad de Ciencias Naturales y Ciencias de la Salud, Universidad de la Patagonia San Juan Bosco, Ruta Nacional 259 Km. 16,41, U9200 Esquel, Chubut, Argentina.

${ }^{3}$ Cátedra de Diversidad Biológica I, Departamento de Diversidad Biológica y Ecología, Facultad de Ciencias Exactas, Físicas y Naturales, Universidad Nacional de Córdoba, Av. Vélez Sarsfield 299, X5000JJC Córdoba, Córdoba, Argentina.
\end{abstract}

\begin{abstract}
Pelissero D.; F. Kuhar, M. Pereyra \& E. Nouhra. 2021. Fungal symbionts of leaf-cutting ants of the genus Acromyrmex in dry forest of central Argentina. Darwiniana, nueva serie 9(1): 162-172.
\end{abstract}

Fungi associated with leaf-cutter ants (LCAs) play a fundamental role in Neotropical ecosystems. The association with fungi allows LCAs to be the dominant herbivores and in this way influence ecological processes. In order to know the taxonomic diversity of fungal symbionts associated with LCAs, ten sampling sites were selected corresponding to the Chaqueño Serrano district and the Algarrobo district. Samples of fungi grown by leaf cutter ants were collected, a part of the fungal material was grown in potato agar culture medium with dextrose and another was stored in a CTAB buffer. Subsequently, amplifications of the ITS1, ITS2 and 5.8S ribosomal gene were carried out. A phylogenetic analysis (Maximum Likelihood and Bayesian) was performed with the sequences. Results show that the leaf cutter ants in the sampled region cultivate symbiotic fungi of two well-differentiated phylogenetic clades (known as A and B). The agar cultures showed a slow growth, with development of globose structures named gongylidia that are grouped together forming staphyla (clusters). Among the ants studied, Acromyrmex striatus is associated with fungi from both clades. In the Espinal forest (Algarrobo district) A. striatus was found associated with clade B fungi (Leucocoprinus sp.) and in the Chaco Serrano (Chaqueño Serrano district) with clade A fungi (Leucocoprinus gongylophorus). While Acromyrmex crassispinus and A. heyeri included in the study exclusively cultivate L. gongylophorus from clade A fungi regardless of the region they inhabit. In addition, the first molecular identification of the fungus L. gongylophorus cultivated by Acromyrmex silvestrii is reported.

Keywords. Acromyrmex; Córdoba; gen 5.8S; ITS1; ITS2; Leucocoprinus.

Resumen. Pelissero D.; F. Kuhar; M. Pereyra \& E. Nouhra. 2021. Hongos simbiontes de hormigas cortadoras de hojas del género Acromyrmex en bosques secos del centro de Argentina. Darwiniana, nueva serie 9(1): 162-172.

Los hongos asociados a hormigas cortadoras de hojas $(\mathrm{HCH})$ juegan un rol fundamental en los ecosistemas Neotropicales. La asociación con hongos permite que las $\mathrm{HCH}$ sean los herbívoros dominantes en esta región, y de esta manera influyan activamente en los procesos ecológicos. A fin de conocer la diversidad taxonómica de simbiontes fúngicos asociados a $\mathrm{HCH}$, se realizó un relevamiento en 10 sitios correspondientes al distrito Chaqueño Serrano dentro de la provincia Chaqueña y al distrito del Algarrobo dentro de la provincia del Espinal. Se recolectaron muestras de hongos cultivados por hormigas cortadoras de hojas, y una parte del material fúngico se cultivó en agar papa con dextrosa y otra se almacenó en buffer CTAB. Se realizaron amplificaciones de la región ITS1, ITS2 y gen 5.8S ribosomal. Con las secuencias se realizaron análisis filogenéticos (Máxima Verosimilitud y Bayesiano). Los resultados muestran que las hormigas cortadoras de hojas en las regiones muestreadas cultivan hongos simbiontes de dos clados (conocidos como A y B) bien diferenciados filogenéticamente. Los cultivos presentaron un crecimiento lento con desarrollo de estructuras globosas llamadas gongilidios, las cuales se agrupan formando estafilos (racimos). 
Entre las hormigas estudiadas, Acromyrmex striatus se asocia con hongos de ambos clados. En el Espinal (distrito del Algarrobo) se encontró a A. striatus asociada con hongos del clado B (Leucocoprinus sp.) y en el Chaco Serrano (distrito Chaqueño Serrano) a hongos del clado A (Leucocoprinus gongylophorus). Mientras que Acromyrmex crassispinus y $A$. heyeri incluidas en el estudio cultivan exclusivamente a $L$. gongylophorus del clado A independientemente de la región que habiten. Además, se reporta la primera identificación molecular del hongo L. gongylophorus cultivado por Acromyrmex silvestrii.

Palabras clave. Acromyrmex; Córdoba; gen 5.8S; ITS1; ITS2; Leucocoprinus.

\section{INTRODUCCIÓN}

Los hongos cumplen diversos roles fundamentales en los ecosistemas naturales que habitan, como saprófitos descomponedores, simbiontes patógenos y mutualistas, y son componentes clave en la dinámica de los suelos por contribuir al ciclado de la materia orgánica y los nutrientes. En particular, los hongos mutualistas propician la nutrición de las plantas y de otros organismos, mientras que los patógenos tienen una relación antagónica con sus hospederos al reducir su fitness (Dighton \& White, 2017).

En América, ciertos hongos se asocian en simbiosis mutualista con hormigas dentro de las cámaras del hormiguero. Las hormigas cortan hojas $\mathrm{y}$ restos vegetales que mezclan con saliva para formar el sustrato de cultivo del hongo y a cambio, utilizan partes del tejido fúngico para satisfacer sus necesidades nutricionales (Hölldobler \& Willson, 1990; Currie et al.,1999; Currie, 2001; Schultz 2020).

Las hormigas cultivadoras se clasifican dentro de la tribu Attini (Hymenoptera; Formicidae; Myrmicinae), junto con otras de diferente hábito recientemente incluidas en esta tribu (Ward et al., 2015). Dentro de la tribu Attini encontramos la subtribu Attina, que comprende cerca de 250 especies de hormigas cultivadoras de hongos, diferenciadas en dos grupos informales que se basan en sus relaciones filogenéticas: Attinas "superiores" (derivadas), con los géneros Atta, Acromyrmex, Amoimyrmex (llamadas Hormigas Cortadoras de Hojas: HCH), Trachymyrmex, Mycetomoellerius, Paratrachymyrmex y Sericomyrmex, y Attinas "inferiores" (basales), grupo que incluye, entre otros géneros, a Cyphomyrmex, Mycetophylax, Mycetosorytis, Myrmicocrypta, Mycocepurus, Apterostigma (Mueller et al., 1998; Ward et al., 2015; Solomon et al., 2019; Cristiano et al., 2020).

Las Attinas son hormigas endémicas de América $\mathrm{y}$ presentan una mayor riqueza de especies en el Neotrópico (Mayhé-Nunes \& Jaffé, 1998). Se estima que el origen de la simbiosis puede haber ocurrido hace unos 60 millones de años (Mueller et al., 2001; Mueller, 2002; Schultz \& Brady, 2008) y existen varias hipótesis sobre este proceso (Mueller et al., 2001; Branstetter et al., 2017). Las hormigas establecen simbiosis nutricional con diferentes familias de hongos; así, la mayoría de los géneros de Attini se asocian con basidiomicetes de la tribu Leucocoprineae de la familia Agaricaceae, mientras que un grupo de Attini inferiores (especies del grupo Apterostigma pilosum) forman simbiosis con hongos de la familia Pterulaceae (Chapela et al., 1994; Mueller et al., 2001; Munkacsi et al., 2004; Schultz, 2020).

Las $\mathrm{HCH}$ poseen un rol ecológico muy importante en los ecosistemas del Neotrópico. Son consideradas los herbívoros dominantes y su efecto es comparable con los grandes ungulados africanos (Hölldobler \& Willson, 1990; Costa et al., 2008). La distribución del material vegetal en los hormigueros y su estructura contribuyen al aporte de nutrientes, a la porosidad y al drenaje en el suelo (Rico Gray \& Oliveira, 2007). Esto favorece la biota en términos de abundancia y composición de plantas al aumentar su fertilidad. Esto último es importante ya que facilita la sucesión, tanto primaria como la de paisajes perturbados (Wirth et al., 2003; Farji-Brener \& Tadey, 2009).

La simbiosis entre hongos y hormigas está vastamente estudiada, ya que el comportamiento forrajero de las hormigas cortadoras (Atta y Acromyrmex) llamó la atención de los primeros naturalistas. Linnaeus en 1758 registró especies de la tribu Attini, en 1804 Fabricius creó el género Atta, y en 1865 Mayr el género Acromyrmex (Weber, 1966). Möller, por otra parte, fue el primero en observar y reportar la formación de basidiomas por parte del simbionte fúngico, $\mathrm{y}$ lo nombró Rozites gongylophora, material que posteriormente fue transferido a Leucocoprinus gongylophorus (Hein) (Mueller et al., 2017). 
El estadio sexual del hongo es infrecuente y sólo se produce cuando la hormiga reina muere y la colonia abandona el cultivo (Mueller, 2002).

Hasta hace poco tiempo, las evidencias mostraban que las $\mathrm{HCH}$ cultivaban sólo una especie fúngica, Leucocoprinus gongylophorus (Leucocoprineae, Agaricales) y las Attini "superiores" no cortadoras (Trachymyrmex, Mycetomoellerius, Paratrachymyrmex y Sericomyrmex) cultivan otra especie cercana (Leucocoprinus sp.). Dicha correspondencia entre hongos y sus cultivares se explicó cómo coevolución debido a la simbiosis obligada de los mismos (Chapela et al., 1994; Mueller et al., 1998; Mueller et al., 2001). Estudios recientes demuestran que las Attini superiores cultivan distintas especies filogenéticas de hongos que se agrupan en dos clados: A (Leucocoprinus gongylophorus) y B (seis especies filogenéticas del género Leucocoprinus, aún no descriptas) (Mueller et al., 2018).

La mayoría de los estudios sobre hongos cultivados por hormigas Attini fueron realizados en Centro y Norteamérica (Mueller et al., 1998; Green et al., 2002; Mehdiabadi et al., 2012). Teniendo en cuenta que estas hormigas son un modelo para estudiar la simbiosis e inferir relaciones evolutivas, es importante contemplar la diversidad presente en todos los ecosistemas, en particular en los del Neotrópico, región donde se habría originado esta simbiosis (Kusnezov, 1963; Fowler, 1983; Mueller et al., 2017).

La región Neotropical está vastamente representada en Argentina por varios tipos de vegetación y áreas fitogeográficas (Cabrera, 1976). Los estudios sobre hongos asociados a $\mathrm{HCH}$ en estas regiones son actualmente escasos (Lugo et al., 2013; Marfetán et al., 2015; Bich et al., 2017; Mueller et al., 2017; Mueller et al., 2018). Un relevamiento reciente de la diversidad fúngica en la región norte de Argentina a partir de muestras de ADN de suelos indicó la existencia de hongos simbiontes de hormigas cultivadoras de hongos no registrados previamente (Nouhra et al., 2018). En Argentina se citan 4 especies de Atta y 29 especies de Acromyrmex, de ellas unas 18 especies de Acromyrmex y 3 especies de Atta para Córdoba (Janicki et al., 2016; Antweb, 2018). Si bien existe en Córdoba un estudio sobre la diversidad de simbiontes fúngicos asociados a estas hormigas, los hongos no fueron caracterizados taxonómicamente (Abril \& Bucher, 2007).
El objetivo de este trabajo es relevar los simbiontes fúngicos asociados a $\mathrm{HCH}$ del género Acromyrmex en dos distritos fitogeográficos dominantes de la provincia de Córdoba.

\section{MATERIALES Y MÉTODOS}

\section{Sitios de estudio:}

El estudio se realizó en la provincia de Córdoba en el dominio Chaqueño de la región Neotropical. Se seleccionaron 10 sitios de los cuales 7 pertenecen al distrito Chaqueño Serrano de la provincia Chaqueña (Chaco Serrano), 2 al distrito del Algarrobo de la provincia del Espinal (Espinal) y 1 sitio en Ciudad Universitaria Córdoba. Este último se incluyó en el Chaco Serrano por la cercanía que presenta con los sitios de esta región y representa un área de transición muy modificada entre las provincias fitogeográficas muestreadas (Cabrera 1976; Giorgis et al., 2017; Cabido et al., 2018; Zeballos et al., 2020) (Fig. 1).

Los muestreos se realizaron en el período comprendido entre noviembre de 2018 a mayo de 2019, en las siguientes ubicaciones; correspondiente al Distrito Chaqueño Serrano (Chaco Serrano):

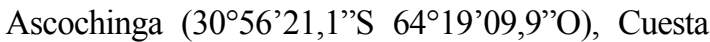
Blanca (31 $\left.{ }^{\circ} 29^{\prime} 42,8^{\prime \prime} \mathrm{S} \quad 64^{\circ} 35^{\prime} 30,7^{\prime \prime} \mathrm{O}\right)$, Camino Altas cumbres $\left(31^{\circ} 35^{\prime} 32,4^{\prime \prime} \mathrm{S} 64^{\circ} 31^{\prime} 34,8^{\prime}\right.$ 'O), La Estancita (31 ${ }^{\circ} 07^{\prime} 04,0^{\prime \prime}$ 'S 64²1'47,4”O), Mendiolaza ( $31^{\circ} 16^{\prime} 18,8^{\prime}$ 'S $\left.64^{\circ} 18^{\prime} 48,8^{\prime \prime} \mathrm{O}\right)$, Camino Falda del

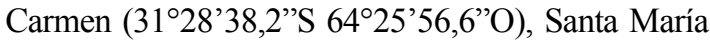
de Punilla (31 ${ }^{\circ} 15^{\prime} 18,38^{\prime \prime}$ 'S 64³6' $15,26^{\circ}$ 'O) y Ciudad Universitaria Córdoba ( $31^{\circ} 26^{\prime} 05,3^{\prime \prime}$ 'S 64¹1'33,2”O) y correspondientes al Distrito del Algarrobo (Espinal): Yucat $\left(32^{\circ} 21^{\prime} 32,2^{\prime \prime} \mathrm{S} \quad 63^{\circ} 26^{\prime} 23,7^{\prime \prime O}\right)$ y Tinoco (3106'08,9'S 6350'44,6”'O) (Fig. 1).

\section{Cultivo y análisis molecular:}

En cada sitio se muestrearon entre 1 y 5 hormigueros de las diferentes especies de $\mathrm{HCH}$ que se encontraron. Las muestras se obtuvieron cavando con una pala hasta acceder a la/las cámara/s conteniendo el/los jardín/es fúngicos, e intentando no destruir completamente el nido. Se extrajo el material fúngico y se aisló "in situ" en placas de Petri (entre 3 y 5 placas por hormiguero) previamente preparadas con medio agar de papa y dextrosa (APD) y un antibiótico (cloranfenicol). Una porción se fijó en fresco en buffer CTAB (Bromuro 

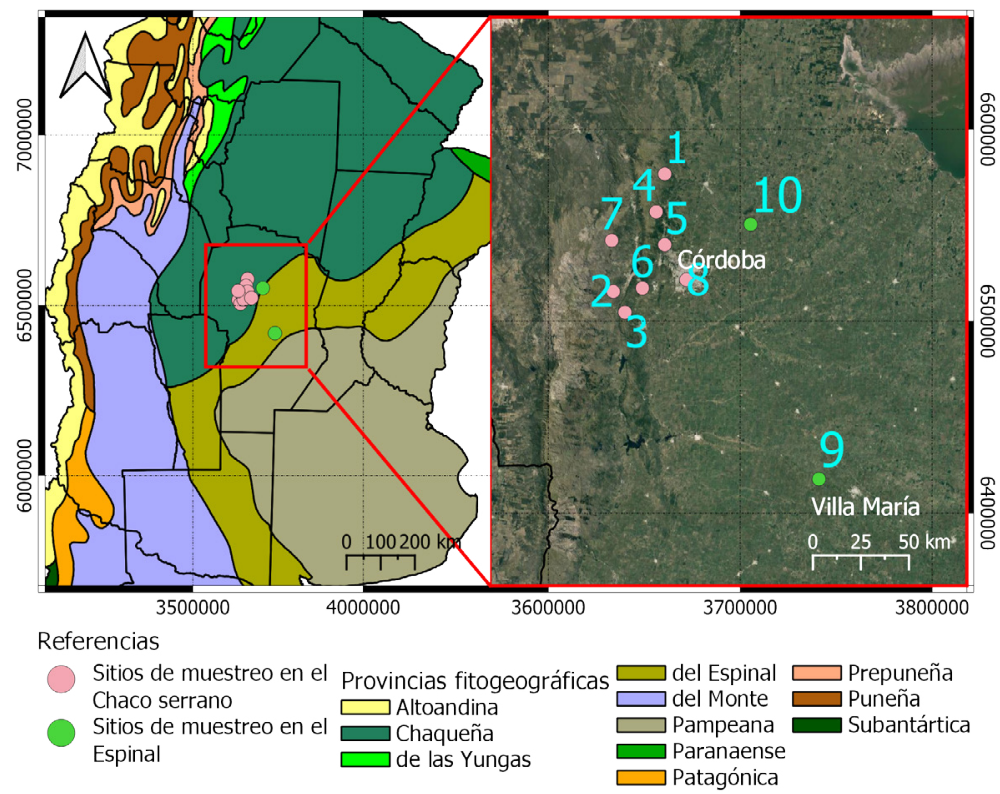

Fig. 1. Sección de las provincias fitogeográficas de Argentina (Cabrera 1976). El rectángulo rojo representa una ampliación de las áreas de muestreo con sus respectivos sitios de muestreo:1) Ascochinga, 2) Cuesta Blanca, 3) Camino Altas cumbres, 4) La Estancita, 5) Mendiolaza, 6) Camino Falda del Carmen, 7) Santa María, 8) Ciudad Universitaria, 9) Yucat, 10) Tinoco. Figura en color en la versión en línea http://www.ojs.darwin.edu.ar/index.php/darwiniana/article/view/950/1219

de cetiltrimetilamonio) para la posterior extracción y análisis de ADN. Para cada aislamiento se realizaron subcultivos a fin de asegurar su pureza y, cuando aparecieron contaminaciones, se realizaron nuevos repiques y aislamientos con el fungicida selectivo benomyl. Los cultivos se mantuvieron en oscuridad a $25^{\circ} \mathrm{C}$ en cámara de cultivo.

El ADN se extrajo de aproximadamente $50 \mathrm{mg}$ de micelio fijado en CTAB de cultivos en APD (Gardes $\&$ Bruns, 1993) y se amplificó la región ITS (ITS15.8-ITS2) mediante PCR utilizando los primers ITS1F y ITS4B (Gardes \& Bruns, 1993; White et al., 1990). Las amplificaciones se enviaron a Macrogen Inc. (Seúl, Corea del Sur) para la purificación y secuenciación del gen ITS, con el primer ITS4, en un equipo ABI 3730XL. Las secuencias así generadas fueron editadas con BioEdit v7.0.5, y de un total de 29 secuencias, 9 estuvieron completas en sus regiones intergénicas (690 a 700 pb) ITS1, ITS2 y el gen 5.8S ribosomal. De las 20 restantes se utilizaron aproximadamente $110 \mathrm{pb}$ que corresponden a la región intergénica ITS2. Las secuencias se compararon con aquellas disponibles en bases de datos mediante la herramienta de búsqueda "Blast" en Genbank (http://www.ncbi.nlm.gov).
Las secuencias se alinearon con el servidor MAFFT 7.0 (Katoh \& Standley, 2013) y con el servidor online del software Gblocks v. 0.91 b (Castresana, 2002) se eliminaron las regiones ambiguamente alineadas y se extrajeron los bloques comparables para el análisis. Se obtuvieron un total de 29 secuencias con sitios comparables. Se seleccionó K2+I como modelo de sustitución de nucleótidos con jModelTest 2.1 (Darriba et al., 2012) usando el criterio de información Akaike (AIC), y así se realizó un análisis de Máxima Verosimilitud en Mega-X (Kumar et al., 2018). Los valores de Bootstrap para el árbol más verosímil se calcularon sobre 1000 repeticiones. El análisis Bayesiano se realizó con MrBayes (Huelsenbeck \& Ronquist, 2001) produciendo 10000000 de generaciones de árboles aleatorios sobre cuatro cadenas simultáneas y muestreando cada mil árboles.

Los cultivos fúngicos se caracterizaron morfológicamente de acuerdo con la bibliografía especializada (e.g. Weber, 1966; Curie, 2001; Miyashira et al., 2010), utilizando un microscopio óptico Carl Zeiss Primo Star. Para ello se realizaron preparaciones microscópicas coloreadas con floxina B (C.I. 45410), tanto de las estructuras fúngicas observadas en cultivos como aquellas extraídas 
directamente de los jardines fúngicos. Las hormigas se identificaron bajo lupa estereoscópica Nikon (SMZ745T), utilizando la clave de Gonçalves (1961) y una clave provisoria proporcionada por el Dr. Andrés F. Sánchez Restrepo. Las fotografías fueron tomadas con cámara AxioCam ERC 5s.

\section{RESULTADOS}

Se analizaron 28 hormigueros, 20 pertenecientes al distrito del Chaco Serrano, 7 pertenecientes al distrito del Algarrobo y 1 en la Ciudad universitaria de Córdoba (UNC, en un área relictual muy modificada de bosque chaqueño) (Tabla 1).

Se encontró que la diversidad taxonómica de los simbiontes fúngicos cultivados por $\mathrm{HCH}$ pertenecen a 2 clados bien diferenciados filogenéticamente (Fig. 2). El clado A (Leucocoprinus gongylophorus) y el clado B (Leucocoprinus sp.). Los hormigueros pertenecen a 4 especies: Acromyrmex crassispinus, A. striatus,
A. heyeri y $A$. silvestrii (Fig. 3 - A, B, C y D). Se encontró que $A$. striatus cultiva hongos de ambos clados, el A en el Chaco Serrano y el B en el Espinal. El resto de las hormigas cultivan cepas del clado A independientemente de la región de la cual provengan.

\section{Posición filogenética de los simbiontes fúngicos}

Las relaciones filogenéticas observadas (Fig. 2) indican que las $\mathrm{HCH}$ se asocian en simbiosis nutricional con cepas de los dos clados antes mencionados. El clado A, con un soporte alto (99/1), representa un grupo monofilético de la especie Leucocoprinus gongylophorus. El clado B con igual soporte (99/1), representaría una especie aún no descrita del género Leucocoprinus.

Las hormigas $A$ crassispinus y $A$ heyeri cultivan siempre a $L$. gongylophorus, independientemente de la región. Acromyrmex striatus cultiva en el Chaco Serrano a L. gongylophorus y en el Espinal a Leucocoprinus sp. La hormiga $A$. silvestrii cultiva a L. gongylophorus en el Chaco Serrano.

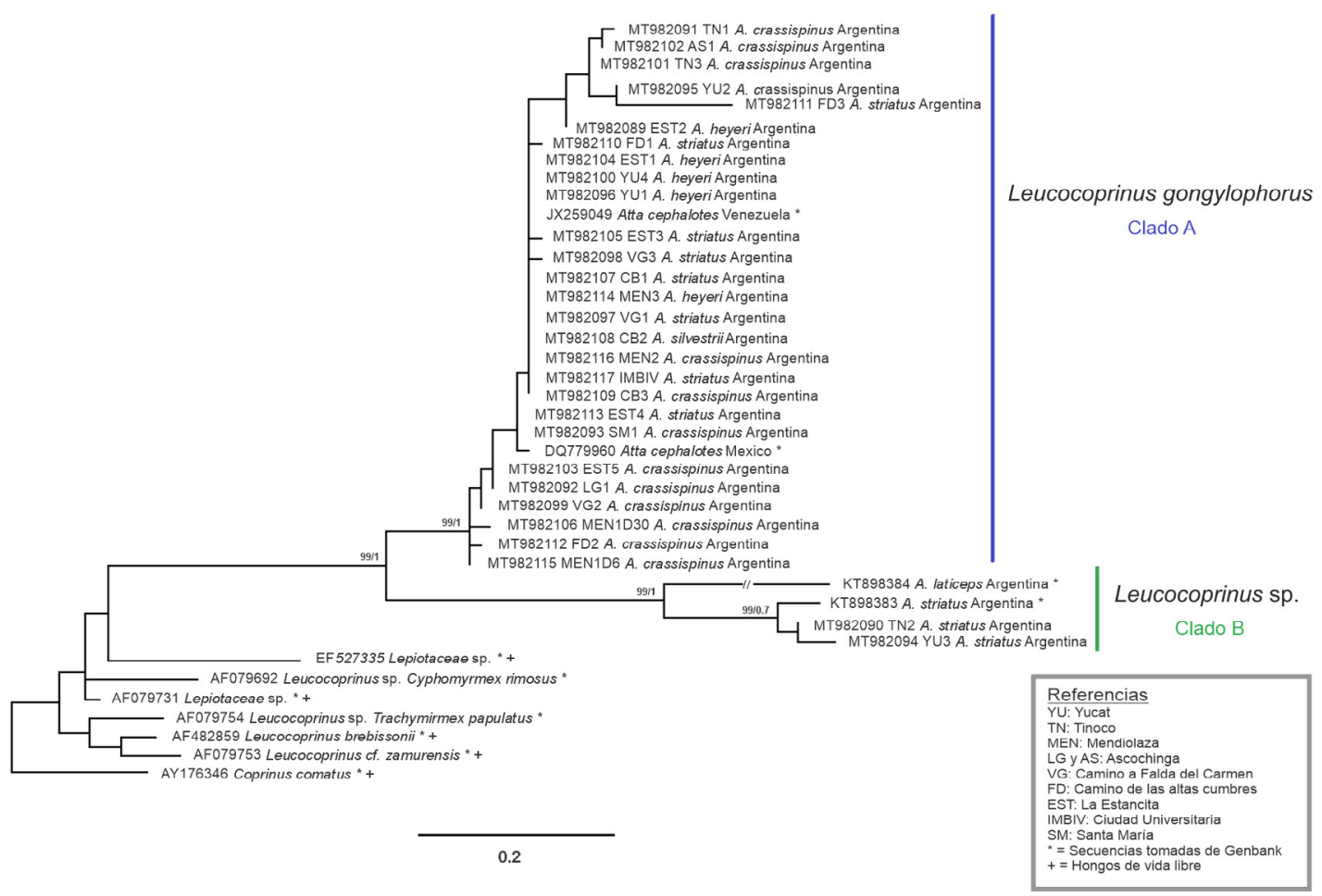

Fig. 2. Árbol filogenético obtenido por Máxima Verosimilitud. Las medidas de soporte son provistas por Bootstrap y Probabilidades Posteriores respectivamente. Coprinus comatus se utilizó como outgroup para enraizar el árbol. Los nombres de los simbiontes cuentan con su código de acceso a Genbank y la especie de hormiga asociada. La barra de escala representa el número esperado de cambios de nucleótidos por sitio. 
Tabla 1. Número de hormigueros por cada sitio muestreado y especie de hormiga asociada.

\begin{tabular}{|c|c|c|c|c|c|}
\hline \multirow{2}{*}{$\begin{array}{l}\text { Región } \\
\text { fitogeográfica }\end{array}$} & \multirow{2}{*}{ Sitio } & \multicolumn{4}{|c|}{ Especie de hormiga } \\
\hline & & A. striatus & A. crassispinus & A. heyeri & A. silvestrii \\
\hline \multirow{8}{*}{ Chaco Serrano } & Mendiolaza & - & 2 & 1 & - \\
\hline & La Estancita & 2 & 1 & 2 & - \\
\hline & Falda del Carmen & 2 & 1 & - & - \\
\hline & Ascochinga & - & 2 & - & - \\
\hline & Camino Altas cumbres & 2 & 1 & - & - \\
\hline & Cuesta Blanca & 1 & 1 & - & 1 \\
\hline & Santa María de Punilla & - & 1 & - & - \\
\hline & Ciudad Universitaria & 1 & - & - & - \\
\hline \multirow{2}{*}{ Espinal } & Tinoco & 1 & 2 & - & - \\
\hline & Yucat & 1 & 1 & 2 & - \\
\hline
\end{tabular}

\section{Caracterización morfológica de cultivos}

Los cultivos del simbionte fúngico se iniciaron en diferentes momentos debido a las fechas de recolecta y a los sucesivos repiques que se realizaron por contaminación. Todos comenzaron presentando una zona central de aspecto algodonoso, donde se observó una elevada densidad de hifas que disminuyeron en abundancia hacia la periferia, apareciendo hifas hialinas solo en los bordes externos del cultivo $(0,5 \mathrm{~cm}$ de diámetro aproximado a los 15 días de cultivo). Al cabo de 30 días (aprox.), cuando los cultivos poseían un tamaño entre 1 y $1,5 \mathrm{~cm}$ de diámetro, las colonias presentaron exudados en forma de gotas hialinas amarillentas sobre la superficie del cultivo y desarrollaron estafilos con forma de copos blanquecinos, formados por agrupaciones de gongilidios (Fig. 4- C y D). Estas estructuras globosas se forman en las terminaciones hifales y se encuentran agrupadas en racimos denominados estafilos (Fig. 4- A y B). Si bien el simbionte fúngico del clado $\mathrm{B}$ presentó estafilos menos evidentes a simple vista, su crecimiento en cultivo fue similar al descrito anteriormente.

En los cultivos de ambos simbiontes (clados A y B), se observaron al microscopio, hifas hialinas cilíndricas, alargadas y delgadas con ramificaciones laterales y en forma de $\mathrm{H}$. con septos simples y desprovistas de fíbulas. El grosor de las hifas en ambos fue de 4-7,5 $\mu \mathrm{m}$. Los gongilidios presentaron variación en su forma a los 120 días de crecimiento en placa. El clado A presentó un grosor promedio de $27 \mu \mathrm{m}$ (rango de $15-37 \mu \mathrm{m}$ ) y un largo promedio de $44 \mu \mathrm{m}$ (rango de 37-50 $\mu \mathrm{m}$ ), de forma ovoide (Fig. 4- B y D).
El clado B presentó un grosor promedio de $33 \mu \mathrm{m}$ (rango de $20-50 \mu \mathrm{m}$ ) y un largo promedio de $37 \mu \mathrm{m}$ (rango de 25-52.5 $\mu \mathrm{m}$ ), de aspecto globular (Fig. 4A y C). En ambos cultivos los gongilidios fueron terminales, laterales y ocasionalmente intercalares.

\section{DISCUSIÓN}

\section{Características de la simbiosis}

En este trabajo, los resultados filogenéticos indican que la diversidad fúngica asociada a las $\mathrm{HCH}$ de los sitios estudiados estaría agrupada en dos clados bien diferenciados del género Leucocoprinus (Agaricaceae). Estos resultados son concordantes con los estudios recientes de Mueller et al. $(2017,2018)$, en los cuales se incluyeron hormigas con distribución en la Sudamérica austral.

Acromyrmex striatus cultivó cepas de ambos clados, el B (Leucocoprinus sp.) tal como lo reportaron Mueller et al. (2018) y el A (Leucocoprinus gongylophorus). La interacción de A. striatus con el simbionte de clado A no está registrada en la bibliografía y dependiendo de la región que habita, A. striatus cultiva uno u otro simbionte fúngico. Otros estudios reportan que, en Brasil, las hormigas cortadoras (Acromyrmex crassispinus, Acromyrmex coronatus y Atta laevigata) también cultivan ambos clados (Mueller et al., 2018). Esto sugiere que existen mecanismos de transferencia de las cepas fúngicas entre Attini superiores cortadoras y no cortadoras, dado que estas últimas se han encontrado con mayor frecuencia asociadas al simbionte fúngico del clado B (Mueller, 1998; Mueller et al., 2017). 

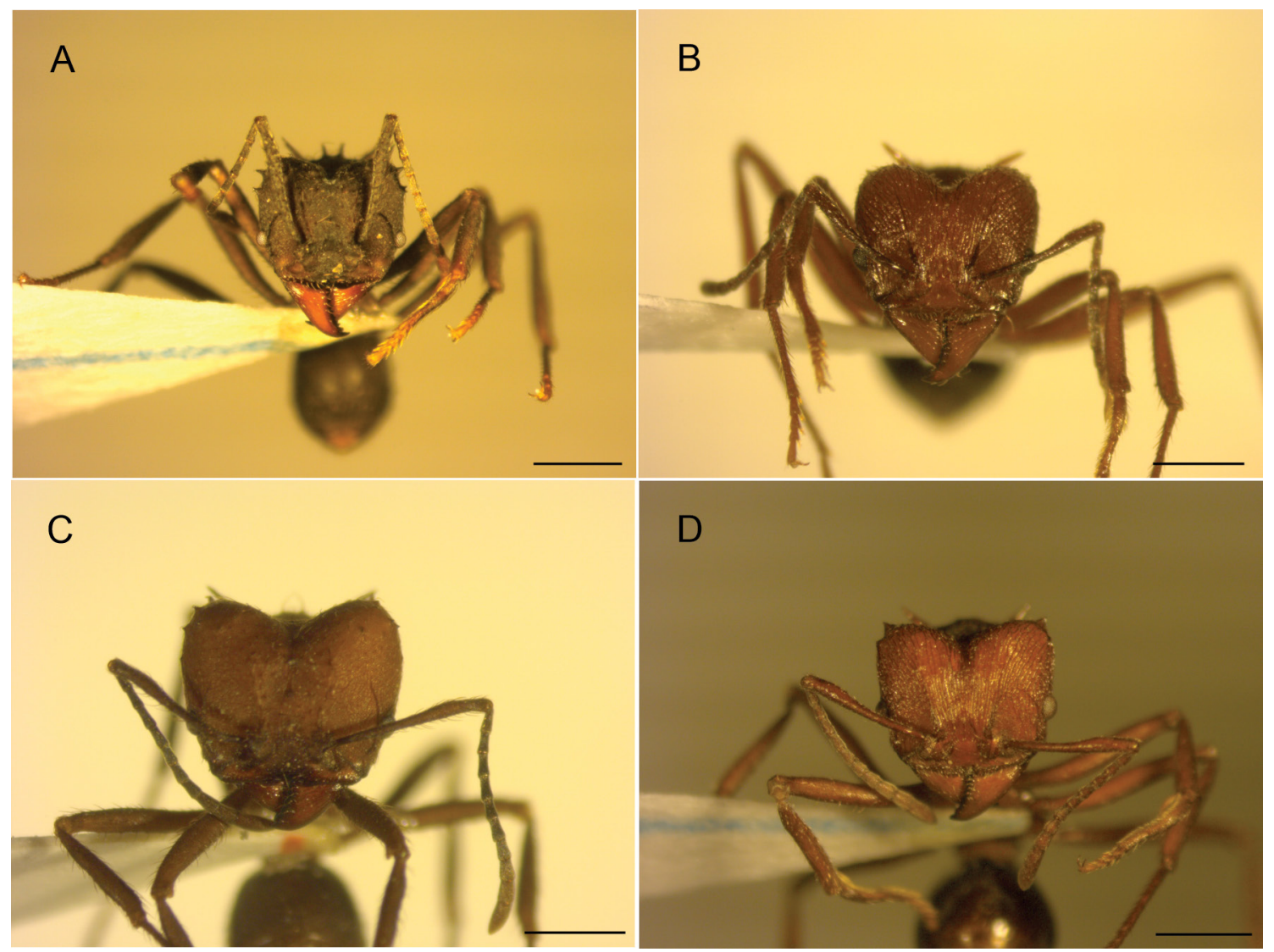

Fig. 3. Vista frontal de hormigas cortadoras de hojas. A, Acromyrmex crassispinus. B, Acromyrmex striatus. C, Acromyrmex heyeri. D, Acromyrmex silvestrii. Escala $=1 \mathrm{~mm}$. Figura en color en la versión en línea http:// www.ojs.darwin.edu.ar/index.php/darwiniana/article/view/950/1219

Las hormigas Acromyrmex crassispinus, $A$. heyeri y A. silvestrii incluidas en nuestro análisis filogenético cultivan cepas del clado A (Leucocoprinus gongylophorus), tal como lo hacen otras especies en Argentina, como A. lobicornis (Lugo et al., 2013) y A. pubescens (Bich et al., 2017). Los resultados de este estudio concuerdan con trabajos previos en la ocurrencia de la asociación de A. heyeri y L. gongylophorus (Pereira et al., 2015).

A diferencia de nuestros resultados, en Brasil se observó que Acromyrmex crassispinus además de cultivar cepas del clado A también lo hace con cepas correspondientes al clado B (Mueller et al., 2017). En este estudio, sin embargo, la hormiga $A$. crassispinus cultiva a $L$. gongylophorus en todos los hormigueros analizados, independiente de la región que se encuentren.
Por otro lado, se analizaron Acromyrmex silvestrii y $A$. striatus que son cercanas filogenéticamente y basales dentro del género (Cristiano et al., 2013). El hormiguero de A. silvestrii incluido en este análisis se encontró en la región Chaqueña Serrana y se asocia con el simbionte fúngico del clado A. La caracterización molecular de este último es nueva para A. silvestrii.

El simbionte fúngico del clado $\mathrm{B}$ representa una especie (o complejo de especies) aún no descrita formalmente, debido a que no se ha recolectado su cuerpo fructífero (basidioma), pero informalmente suele denominarse como "Trachymyces" (e.g. Seal \& Mueller, 2014) ya que siempre se lo encontró asociado a hormigas no cortadoras del género Trachymyrmex. 


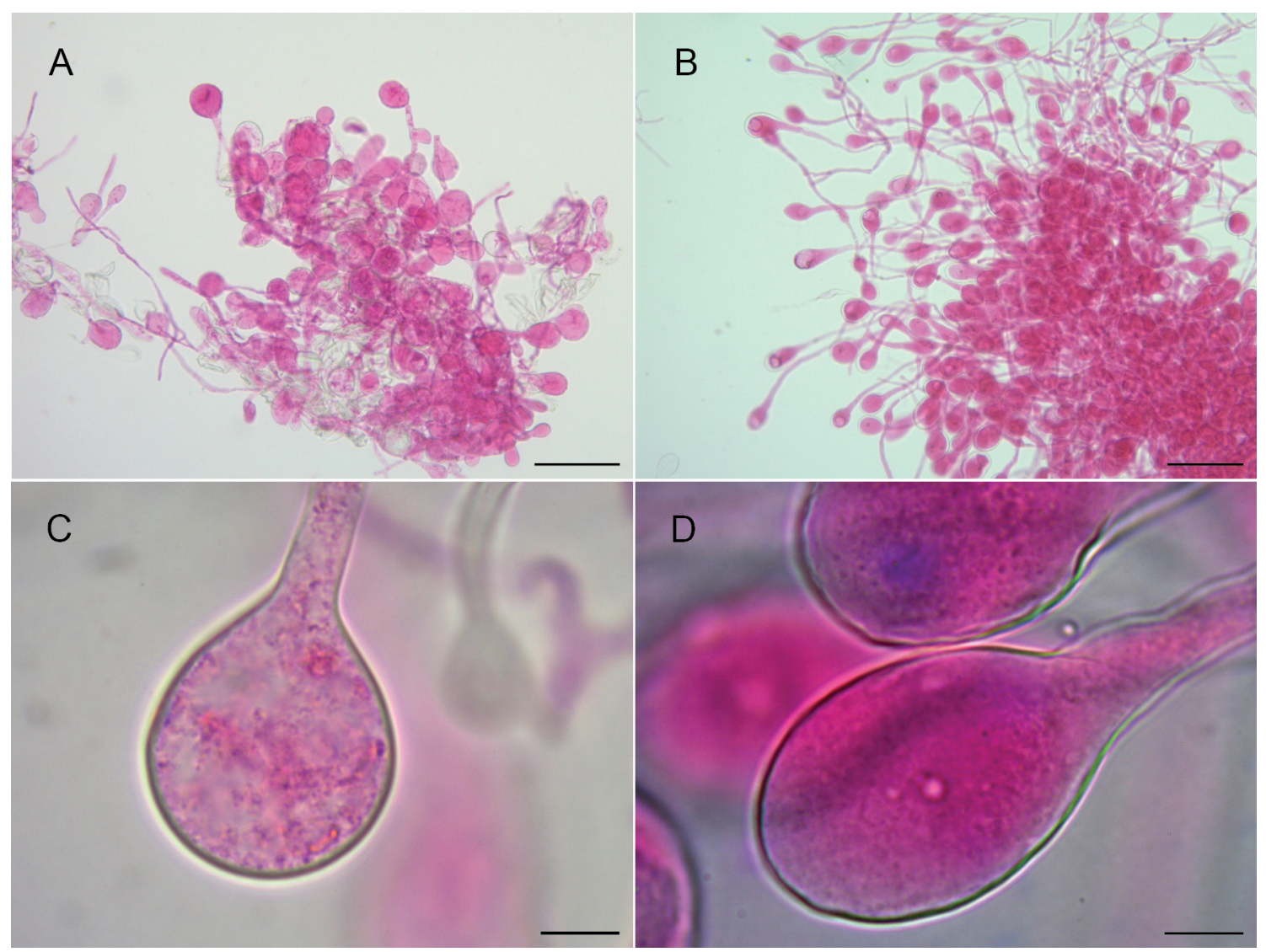

Fig. 4. Estafilos y gongilidios respectivamente en cultivo teñidos con floxina. A-C, Leucocoprinus sp. (clado B). B-D, Leucocoprinus gongylophorus (clado A). Escalas: A y B $=100 \mu \mathrm{m}, \mathrm{C}$ y D $=10 \mu \mathrm{m}$. Figura en color en la versión en línea http://www.ojs.darwin.edu.ar/index.php/darwiniana/article/view/950/1219

Los resultados de este estudio y la literatura sugieren que existe transferencias de simbiontes entre poblaciones de hormigas a nivel intra e interespecíficas (Mikheyev et al., 2006; Mueller et al., 2011a, 2017; Howe et al., 2018). Se ha observado que los mecanismos de transferencia pueden ser mediados por el hurto del simbionte, la adopción de jardines fúngicos abandonados o la usurpación de hormigueros por parte de las reinas fundadoras (Howe et al., 2018). Por esto, se infiere que la asociación entre las hormigas y hongos dependerá de las cepas fúngicas que se encuentren en el área que habitan las hormigas, la frecuencia en la que se den las transferencias $\mathrm{y}$ de que la combinación que se encuentre espacialmente sea favorecida por las fuerzas de selección (Mueller et al., 1998, 2011b).
Esto último se debe a que se ha observado que algunas especies de hormigas no sobreviven cuando se les intercambia el simbionte fúngico (Seal \& Mueller, 2014).

Las evidencias actuales y estos resultados sugieren que la coevolución apareada propuesta (Chapela et al., 1994; Mueller et al., 1998, 2001; Schultz \& Brady, 2008) sería de tipo difuso, y que los mecanismos de intercambio de simbiontes serían muy frecuentes (Howe et al., 2018). Por ello, se piensa que la relación simbiótica especialista de hormigas que cultivan un único simbionte fúngico (Mikheyev et al., 2006) no sería tal, sino que diversos hongos serían cultivados por hormigas diferentes, como resultado de la transferencia de cultivares entre especies y géneros diversos de hormigas cultivadoras (Mueller et al., 2018). 


\section{Caracterización morfológica de cultivos}

Los cultivos de ambos simbiontes son morfológicamente similares, presentando el desarrollo de gongilidios reunidos en estafilos. Si bien, la forma de estos fue diferente en los cultivos analizados, no se puede concluir que el carácter se mantenga constante dado que el crecimiento para cada cultivo fue medido en distintos momentos, como consecuencia de las sucesivas contaminaciones y repiques. Sin embargo, se observó que los estafilos eran menos abundantes en los cultivos del clado B. Esta observación concuerda con los análisis realizados por De Fine Licht et al. (2014) donde el clado B presentó menor densidad de estafilos por unidad de superficie.

\section{CONCLUSIONES}

Los hongos simbiontes de hormigas cortadoras de hojas en la región Chaqueña Serrana y del Espinal en Córdoba pertenecen a dos especies fúngicas diferentes, Leucocoprinus gongylophorus (clado A) y una especie no descripta del mismo género (clado B).

En cuanto a las hormigas, Acromyrmex striatus cultivó en los sitios de Espinal (Distrito del Algarrobo) el clado B, y en los sitios del Chaco Serrano (Distrito Serrano) el clado A. Por otra parte, Acromyrmex crassispinus y A. heyeri cultivaron en ambas regiones cepas fúngicas del clado $\mathrm{A}$.

Se describe por primera vez a nivel morfológico y molecular (ITS) a Leucocoprinus gongylophorus en asociación con $A$. silvestrii para la región Neotropical. En el área de estudio, la relación simbiótica coevolutiva entre hongos y hormigas sería difusa y no altamente especializada. Estudios adicionales que incluyan sitios y simbiontes alternativos, podrían proporcionar datos adicionales sobre esta asociación.

\section{AGRADECIMIENTOS}

A los integrantes del Laboratorio de Micología y del Laboratorio de Biología Molecular del IMBIVCONICET por el apoyo, ayuda y sugerencias. A la SECYT de la Universidad Nacional de Córdoba por el financiamiento para viajes y la generación de secuencias. Por último, al Consejo Interuniversitario Nacional por contribuir a la formación de David Pelissero al otorgar una Beca Estímulo a las Vocaciones Científicas.

\section{BIBLIOGRAFÍA}

Abril, A. B. \& E. H. Bucher. 2007. Genetic diversity of fungi occurring in nests of three Acromyrmex leaf-cutting ant species from Córdoba, Argentina. Microbial ecology 54(3): 417-423. DOI: http://dx.doi.org/10.1007/s00248-007-9252-z

AntWeb. Disponible en: https://www.antweb.org/taxonomicPage. do rank $=$ genus $\&$ country Name $=$ Argentina $\&$ images $=$ true (Acceso 18 junio 2018).

Bich, G. A.; M. L. Castrillo, L. L. Villalba \& P. D. Zapata. 2017. Isolation of the symbiotic fungus of Acromyrmex pubescens and phylogeny of Leucoagaricus gongylophorus from leafcutting ants. Saudi journal of biological sciences 24(4): 851856. DOI: http://dx.doi.org/10.1016/j.sjbs.2016.05.010

Branstetter, M. G.; A. Ješovnik, J. Sosa-Calvo, M. W. Lloyd, B. C. Faircloth, S. G. Brady \& T. R. Schultz. 2017. Dry habitats were crucibles of domestication in the evolution of agriculture in ants. Proceedings of the Royal Society B: Biological Sciences, 284(1852): 20170095. DOI: http:// dx.doi.org/10.1098/rspb.2017.0095

Cabido, M.; S. R. Zeballos, M. Zak, M. L. Carranza, M. A. Giorgis, J. J. Cantero, \& A. T. Acosta. 2018. Native woody vegetation in central Argentina: Classification of Chaco and Espinal forests. Applied Vegetation Science 21(2): 298-311. DOI: http://dx.doi.org/10.1111/avsc. 12369

Cabrera, A. L. 1976. Regiones fitogeográficas argentinas. Enciclopedia argentina de agricultura y jardinería, tomo 2, fasc. 1. Buenos Aires: Acme.

Castresana, J. 2002. Gblocks, v. 0.91 b. versión online disponible en: http://molevol.cmima.csic.es/castresana/ Gblocks_server.html (Acceso 2 de febrero 2020).

Chapela, I. H.; S. A. Rehner, T. R. Schultz \& U. G. Mueller. 1994. Evolutionary history of the symbiosis between fungusgrowing ants and their fungi. Science 266(5191): 1691-1694. DOI: http://dx.doi.org/10.1126/science.266.5191.1691

Costa, A. N.; H. L. Vasconcelos, E. H. Vieira-Neto \& E. M. Bruna. 2008. Do herbivores exert top-down effects in Neotropical savannas? Estimates of biomass consumption by leaf-cutter ants. Journal of Vegetation Science 19(6): 849-854. DOI: http://dx.doi.org/10.3170/2008-8-18461

Cristiano, M. P.; D. C. Cardoso \& T. M. Fernandes-Salomão. 2013. Cytogenetic and molecular analyses reveal a divergence between Acromyrmex striatus (Roger, 1863) and other congeneric species: taxonomic implications. PLoS One 8: 3. DOI: http://dx.doi.org/10.1371/journal.pone.0059784

Cristiano, M. P.; D. C. Cardoso, V. E. Sandoval, Gómez \& F. C. Simões, Gomes. 2020. Amoimyrmex Cristiano, Cardoso \& Sandoval, gen. nov. (Hymenoptera: Formicidae): a new genus of leaf-cutting ants revealed by multilocus molecular 


\section{PELISSERO ET AL. Hongos simbiontes de hormigas cortadoras de hojas}

phylogenetic and morphological analyses. Austral Entomology 59(4): 643-676.

Currie, C. R.; J. A. Scott, R. C. Summerbell \& D. Malloch. 1999. Fungus-growing ants use antibiotic-producing bacteria to control garden parasites. Nature 398(6729): 701. DOI: http://dx.doi.org/10.1038/19519

Currie, C. R. 2001. A community of ants, fungi, and bacteria: a multilateral approach to studying symbiosis. Annual Reviews in Microbiology 55(1): 357-380. DOI: http://dx.doi. org/10.1146/annurev.micro.55.1.357

Darriba, D.; G. L. Taboada, R. Doallo \& D. Posada. 2012. jModelTest 2: more models, new heuristics and parallel computing. Nature methods 9(8): 772. DOI: http://dx.doi. org/10.1038/nmeth.2109

De Fine Licht, H. H.; J. J. Boomsma \& A. Tunlid. 2014. Symbiotic adaptations in the fungal cultivar of leaf-cutting ants. Nature Communications 5: 5675. DOI: http://dx.doi. org/10.1038/ncomms6675

Dighton, J. \& J. F. White. 2017. The fungal community: its organization and role in the ecosystem, $4^{\mathrm{a}}$ edición, 32 vols. Boca Ratón, Florida: CRC Press.

Farji-Brener, A. G. \& M. Tadey. 2009. Contributions of leafcutting ants to soil fertility: causes and consequences. In Soil Fertility, D. P. Lucero \& J. E. Boggs eds, Chapter 6, 81-91. New York: Nova Science Publishers.

Fowler, H. G. 1983. Latitudinal gradients and diversity of the leaf-cutting ants (Atta and Acromyrmex) (Hymenoptera: Formicidae). Revista de Biología Tropical 31(2): 213-216.

Gardes, M. \& T. D. Bruns. 1993. ITS primers with enhanced specificity for basidiomycetes - application to the identification of mycorrhizae and rusts. Molecular ecology 2(2): 113-118. DOI: http://dx.doi.org/10.1111/j.1365-294X.1993.tb00005.x

Giorgis, M. A.; A. M. Cingolani, D. E. Gurvich, P. A. Tecco, J. Chiapella, F. Chiarini \& M. Cabido. 2017. Changes in floristic composition and physiognomy are decoupled along elevation gradients in central Argentina. Applied Vegetation Science 20(4): 558-571. DOI: http://dx.doi.org/10.1111/avsc.12324

Gonçalves, C. R. 1961. O gênero Acromyrmex no Brasil (Hym. Formicidae). Studia Entomologica 4(1-4): 113-180.

Green, A. M.; U. G. Mueller \& R. M. M. Adams. 2002. Extensive exchange of fungal cultivars between sympatric species of fungus-growing ants. Molecular Ecology 11(2): 191-195.

Hölldobler, B. \& E. O. Wilson. 1990. The ants. Cambridge, Massachusetts: Harvard University Press.

Howe, J.; M. Schiøtt \& J. J. Boomsma. 2018. Horizontal partner exchange does not preclude stable mutualism in fungusgrowing ants. Behavioral Ecology 30(2): 372-382. DOI: http://dx.doi.org/10.1093/beheco/ary 176

Huelsenbeck, J. P. \& F. Ronquist. 2001. MRBAYES: Bayesian inference of phylogenetic trees. Bioinformatics, 17(8): 754755.DOI: http://dx.doi.org/10.1093/bioinformatics/17.8.754

Janicki, J.; N. Narula, M. Ziegler, B. Guénard \& E. P. Economo. 2016. Visualizing and interacting with largevolume biodiversity data using client-server web-mapping applications: The design and implementation of antmaps. org. Ecological Informatics 32: 185-193. DOI: http://dx.doi. org/10.1016/j.ecoinf.2016.02.006

Katoh, K. \& D. M. Standley. 2013. MAFFT multiple sequence alignment software version 7: improvements in performance and usability. Molecular biology and evolution 30(4): 772780. DOI: http://dx.doi.org/10.1093/molbev/mst010

Kumar, S.; G. Stecher, M. Li, C. Knyaz \& K. Tamura. 2018. MEGA X: molecular evolutionary genetics analysis across computing platforms. Molecular biology and evolution 35(6): 1547-1549. DOI: http://dx.doi.org/10.1093/molbev/msy096

Kusnezov, N. 1963. Zoogeografía de las hormigas en Sudamérica. Acta Zoológica Lilloana 19: 25-186.

Lugo, M. A.; E. M. Crespo, M. Cafaro \& L. Jofre. 2013. Hongos asociados con dos poblaciones de Acromyrmex lobicornis (Formicidae) de San Luis, Argentina. Boletín de la Sociedad Argentina de Botánica 48(1): 5-15.

Marfetán, J. A.; A. I. Romero \& P. J. Folgarait. 2015. Pathogenic interaction between Escovopsis weberi and Leucoagaricus sp.: mechanisms involved and virulence levels. Fungal ecology 17: 52-61. DOI: http://dx.doi.org/10.1016/j.funeco.2015.04.002

Mayhé-Nunes, A. J. \& K. Jaffé. 1998. On the biogeography of Attini (Hymenoptera: Formicidae). Ecotropicos 11(1): 45-54.

Mehdiabadi, N. J.; U. G. Mueller, S. G. Brady, A. G. Himler \& T. R. Schultz. 2012. Symbiont fidelity and the origin of species in fungus-growing ants. Nature Communications 3: 840. DOI: http://dx.doi.org/10.1038/ncomms 1844

Mikheyev, A. S.; U. G. Mueller \& P. Abbot. 2006. Cryptic sex and many-to-one coevolution in the fungus-growing ant symbiosis. Proceedings of the National Academy of Sciences 103(28): 10702-10706. DOI: http://dx.doi.org/10.1073/ pnas.0601441103

Miyashira, C. H.; D. G. Tanigushi, A. M. Gugliotta \& D. Y. A. C. Santos. 2010. Comparison of radial growth rate of the mutualistic fungus of Atta sexdens rubropilosa Forel in two culture media. Brazilian Journal of Microbiology 41(2): 506-511.

Mueller, U. G.; S. A. Rehner \& T. R. Schultz. 1998. The evolution of agriculture in ants. Science 281(5385): 2034-2038. DOI: http://dx.doi.org/10.1126/science.281.5385.2034

Mueller, U. G.; T. R. Schultz, C. R Currie, R. M. Adams \& D. Malloch. 2001. The origin of the attine ant-fungus mutualism. The Quarterly Review of Biology 76(2): 169197. DOI: http://dx.doi.org/10.1086/393867 
Mueller, U. G. 2002. Ant versus fungus versus mutualism: antcultivar conflict and the deconstruction of the attine antfungus symbiosis. The American Naturalist 160(4): 67-98. DOI: http://dx.doi.org/10.1086/342084

Mueller, U. G.; A. S. Mikheyev, S. E. Solomon \& M. Cooper. 2011a. Frontier mutualism: coevolutionary patterns at the northern range limit of the leaf-cutter ant-fungus symbiosis. Proceedings of the Royal Society B: Biological Sciences 278(1721): 3050-3059. DOI: http://dx.doi.org/10.1098/ rspb.2011.0125

Mueller, U. G.; A. S. Mikheyev, E. Hong, R. Sen, D. L. Warren, S. E. Solomon, H. D. Ishak, M. Cooper, J. L. Miller, K. A. Shaffer \& T. E. Juenger. 2011b. Evolution of cold-tolerant fungal symbionts permits winter fungiculture by leafcutter ants at the northern frontier of a tropical ant-fungus symbiosis. Proceedings of the National Academy of Sciences 108(10): 4053-4056. DOI: http://dx.doi.org/10.1073/pnas.1015806108

Mueller, U. G.; H. D. Ishak, S. M. Bruschi, C. C. Smith, J. J. Herman, S. E. Solomon, A. S. Mikheyev, C. Rabeling, J. J. Scott, M. Cooper, A. Rodrigues, A. Ortiz, C. R. F. Brandão, J. E. Lattke, F. C. Pagnocca, S. A. Rehner, T. R. Schultz, H. L. Vasconcelos, R. M. M. Adams, M. Bollazzi, R. M. Clark, A. G. Himler, J. S. LaPolla, I. R. Leal, R. A. Johnson, F. Roces, J. Sosa-Calvo, R. Wirth \& M. Bacci Jr. 2017. Biogeography of mutualistic fungi cultivated by leafcutter ants. Molecular ecology 26(24): 6921-6937. DOI: http:// dx.doi.org/10.1111/mec.14431

Mueller, U. G.; M. R. Kardish, H. D. Ishak, A. M. Wright, S. E. Solomon, S. M. Bruschi, A. L. Carlson \& M. Bacci Jr. 2018. Phylogenetic patterns of ant-fungus associations indicate that farming strategies, not only a superior fungal cultivar, explain the ecological success of leafcutter ants. Molecular ecology 27(10): 2414-2434. DOI: http://dx.doi.org/10.1111/ mec. 14588

Munkacsi, A. B.; J. J. Pan, P. Villesen, U. G. Mueller, M. Blackwell \& D. J. McLaughlin. 2004. Convergent coevolution in the domestication of coral mushrooms by fungus-growing ants. Proceedings of the Royal Society of London B: Biological Sciences 271(1550): 1777-1782. DOI: http://dx.doi.org/10.1098/rspb.2004.2759

Nouhra, E.; F. Soteras, N. Pastor \& J. Geml. 2018. Richness, species composition and functional groups in Agaricomycetes communities along a vegetation and elevational gradient in the Andean Yungas of Argentina. Biodiversity and Conservation 27(8): 1849-1871. DOI: https://doi.org/10.1007/s10531-018-1512-3

Pereira, D. I. B.; S. D. A Botton, M. I. D. Azevedo, D. U. Monteiro, C. Weiblen, V. S. Machado, M. Guimarães Donatti \& A. E. Loeck. 2015. Isolation and molecular characterization of symbiotic fungus from Acromyrmex ambiguus and Acromyrmex heyeri ants of Rio Grande do Sul State, Brazil. Ciência Rural 45(7): 1256-1261. DOI: http:// dx.doi.org/10.1590/0103-8478cr20141064

Rico-Gray, V. \& P. S. Oliveira. 2007. The ecology and evolution of ant-plant interactions. Chicago Illinois: University of Chicago Press. DOI: http://dx.doi.org/10.7208/ chicago/9780226713540.001.0001

Schultz, T. R. \& S. G. Brady. 2008. Major evolutionary transitions in ant agriculture. Proceedings of the National Academy of Sciences 105(14): 5435-5440. DOI: http:// dx.doi.org/10.1073/pnas.0711024105

Schultz, T. R. 2020. Fungus-Farming Ants (Attini in Part). En Encyclopedia of Social Insects. Springer International Publishing. DOI: http://dx.doi.org/10.1007/978-3-31990306-4_46-1

Seal, J. N. \& U. G. Mueller. 2014. Instability of novel antfungal associations constrains horizontal exchange of fungal symbionts. Evolutionary Ecology 28(1): 157-176. DOI: http://dx.doi.org/10.1007/s10682-013-9665-8

Solomon, S. E.; C. Rabeling, J. Sosa-Calvo, C. T. Lopes, A. Rodrigues, H. L. Vasconcelos, M. Bacci Jr, U. G. Mueller \& T. R. Schultz. 2019. The molecular phylogenetics of Trachymyrmex Forel ants and their fungal cultivars provide insights into the origin and coevolutionary history of 'higher-attine' ant agriculture. Systematic Entomology, 44(4); 939-956. DOI: http://dx.doi.org/10.1111/syen.12370

Ward, P. S.; S. G. Brady, B. L. Fisher \& T. R. Schultz. 2015. The evolution of myrmicine ants: phylogeny and biogeography of a hyperdiverse ant clade (Hymenoptera: Formicidae). Systematic Entomology 40(1): 61-81. DOI: http://dx.doi. org/10.1111/syen.12090

Weber, N.A. 1966. Fungus-growing ants. Science 153(3736): 587604. DOI: http://dx.doi.org/10.1126/science.153.3736.587

White, T. J.; T. Bruns, S. J. W. T. Lee \& J. Taylor. 1990. Amplification and direct sequencing of fungal ribosomal RNA genes for phylogenetics. PCR protocols: a guide to methods and applications 18(1): 315-322. DOI: http:// dx.doi.org/10.1016/B978-0-12-372180-8.50042-1

Wirth, R.; H. Herz, R. J. Ryel, W. Beyschlag \& B. Hölldobler. 2003. Herbivory of leaf-cutting ants: a case study on Atta colombica in the tropical rainforest of Panama, Ecological Studies, 164 Vol. Berlin, Heidelberg, New York: Springer.

Zeballos, S. R.; M. A. Giorgis, M. R. Cabido, A. T. R. Acosta, M. D. R. Iglesias \& J. J. Cantero. 2020. The lowland seasonally dry subtropical forests in central Argentina: vegetation types and a call for conservation. Vegetation Classification and Survey 1: 87-102. DOI: https://doi. org/10.3897/VCS/2020/38013 